\title{
MONOLAYER CHARACTERISTICS OF SOME MULTINUCLEATE PHTHALOCYANINES*
}

J.-H. KIM, T. M. COTTON AND R. A. UPHAUS

Department of Chemistry, University of Nebraska, Lincoln, NE 68588-0304 (U.S.A.)

C. C. LEZNOFF

Department of Chemistry, York University, Downsview, Ontario M3J IP3 (Canada)

Surface pressure-area isotherms were established for a series of seven derivatized phthalocyanine compounds containing from one to four covalently linked macrocycles. Good solubility in spreading solvents was conferred by the presence of four neopentoxyl groups per macrocycle. All compounds studied formed strong, stable monolayers with high breaking pressures (about $50 \mathrm{~m} \mathrm{~N} \mathrm{~m}^{-1}$ or greater). Observed surface pressure-area isotherms were of the most simple kind, with no indication of phase transitions or discontinuities, with one exception. Minimal molecular areas were seen at lowest compression rates and higher temperatures, indicating that these systems have a large number of configurational states which are not capable of restructuring quickly into a compact monolayer of highest density. For some compounds it appears that orientation on the water surface can be essentially flat. Most previously studied phthalocyanine monolayers suggest that the macrocycles tend always to orient edge-on at some acute angle to the water surface because of the very strong cofacial energies of interaction. With other compounds of this group, it is evident that the macrocycles can assume orientations either parallel or perpendicular to the surface, others having intermediate orientations as well. In addition, one multinucleate compound probably assumes a bilayer structure at high surface pressures. The range of orientations available in these systems should appreciably extend the utility of phthalocyanines in monolayers transferred by the Langmuir-Blodgett technique. The present study indicates that a more complete exploitation of the possibilities of organic synthesis can result in a more varied monolayer behavior than seen hitherto.

\section{INTRODUCTION}

The unique optical and electrical properties of phthalocyanine ( $\mathrm{Pc}$ ), as well as its remarkable chemical stability, have stimulated many monolayer studies having the ultimate objective of incorporating monolayers of $\mathrm{Pc}$ as the functional element

* Paper presented at the Third International Conference on Langmuir-Blodgett Films, Göttingen, F.R.G., July 26-31, 1987. 
in various sensors or other devices. The parent, underivatized molecule is intractable and insoluble in all common solvents. Derivatized species must therefore be used for monolayer formation, while maintaining as completely as possible the intrinsic character of the parent compound. Thus, the tetra-t-butyl derivative has been extensively studied ${ }^{1}$. However, even in this compound the strong cofacial bonding interactions may not allow disaggregation to the point where individual molecules can be said to be present in the traditional context of spread monolayers.

Kuhn, Möbius and coworkers ${ }^{2}$ were the first to demonstrate the great potential afforded by the modulation of specific monolayer properties through subtle modification of molecular architecture. This fruitful mode of attack is just beginning to be applied to Pc structures intended for monolayer formation (cf. ref. 3 and citations therein). The recent preparation of novel Pc systems by Leznoff et al. ${ }^{4}$ provides an interesting new group of Pc compounds having possible application to monolayer constructions. They consist of single or multiple macrocycles connected by bridging chains of various lengths (Fig. 1). The neopentoxyl substituent groups provide high solubility in common spreading solvents such as chloroform. The variation in the linking of macrocycles provides the possibility for a great range of assumable configurational states in spread monolayers.

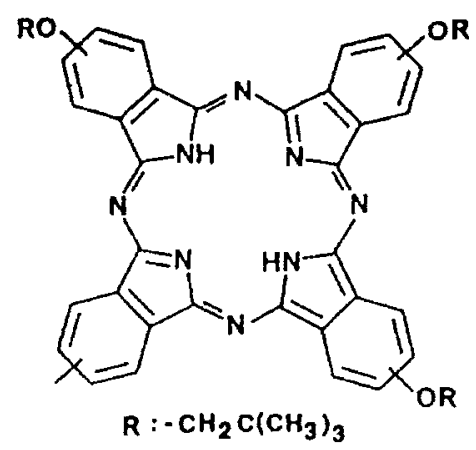

Fig. 1. Pc structures.

\begin{tabular}{ll}
\hline Designation & Structure \\
\hline SG-A & $\mathrm{Pc}-\mathrm{OR}$ \\
SG-B & $\mathrm{Pc}-\mathrm{Pc}$ \\
SG-25 & $\mathrm{Pc}-\mathrm{O}-\mathrm{Pc}$ \\
SG-35 & $\mathrm{Pc}-\mathrm{CH}-\mathrm{CH}_{2}-\mathrm{Pc}$ \\
SG-C & $\mathrm{Pc}-\mathrm{O}-\mathrm{CH}_{2} / \mathrm{CH}_{3}$ \\
& \\
& $\mathrm{Pc}-\mathrm{O}-\mathrm{CH}_{2} \mathrm{CH}_{2} \mathrm{CH}_{3}$ \\
SG-26R & $\mathrm{Pc}-\mathrm{O}-\mathrm{Pc}-\mathrm{O}-\mathrm{Pc}$ \\
SG-tet & $\mathrm{Pc}-\mathrm{O}-\mathrm{CH}_{2} \backslash \mathrm{CH}_{2}-\mathrm{O}-\mathrm{Pc}$ \\
& $\mathrm{Pc}-\mathrm{O}-\mathrm{CH}_{2} \mathrm{CH}_{2}-\mathrm{O}-\mathrm{Pc}$ \\
\hline
\end{tabular}


Apparent molecular areas as determined from surface isotherms are not sufficient evidence for the existence of a specific orientational state, for which other techniques must be used. However, the suggested orientations for the present systems are consistent with the apparent determined areas.

\section{EXPERIMENTAL DETAILS}

The preparation of all compounds was as described in the literature 4 Chloroform was used as the spreading solvent after purification in an alumina column and addition of $1 \%$ ethanol.

Surface isotherms were determined with a Wilhelmy filter paper sensor and a thermostated, automated Teflon Langmuir trough. Area and surface tension data were digitized and stored in a dedicated computer. All measurements were made on systems spread on water of near-neutral $\mathrm{pH}$ which was previously subjected to deionization, reverse osmosis treatment and final distillation in an all glass system.

\section{RESULTS AND DISCUSSION}

All the compounds of this series formed strong monolayers with collapse pressures of about $50 \mathrm{mN} \mathrm{m}^{-1}$ or greater. Isotherms were highly reproducible to within several per cent, were of simple character and, with one exception, lacked plateaux or indications of phase transitions. The apparent areas per molecule as determined from the surface isotherms are summarized in Table $I$. The salient features of the isotherms of the individual compounds are summarized as follows.

TABLE I

MOLECULAR AREAS OF MULTINUClEATE PHTHALOCYANINES

\begin{tabular}{llll}
\hline Compound & $\begin{array}{l}\text { Areaper } \\
\text { molecule }\left(\AA^{2}\right)\end{array}$ & $\begin{array}{l}\text { Temperature } \\
\left({ }^{\circ} \mathrm{C}\right)\end{array}$ & $\begin{array}{l}\text { Compression rate } \\
\left(\AA^{2} \mathbf{m o l}^{-1} \mathbf{m i n}^{-1}\right)\end{array}$ \\
\hline SG-A & 100 & 22 & 30 \\
& 80 & 22 & 10 \\
SG-B & 155 & 22 & 85 \\
& 125 & 22 & 20 \\
SG-C & 288 & 13 & 100 \\
& 246 & 22 & 100 \\
SG-25 & 242 & 32 & 100 \\
SG-26R & 105 & 22 & 55 \\
SG-35 & 338 & 22 & 85 \\
SG-tet & 60 & 22 & 30 \\
& 192 & 22 & 50 \\
\hline
\end{tabular}

\section{1. $S G-A$}

The apparent molecular area of this compound was found to vary somewhat as a function of the compression rate, the lowest compression rate producing a value of $80 \AA^{2}$. This indicates that the structuring of individual molecules into the most dense 
packing cannot occur at high rates of compression. At the maximum packing density the neopentoxyl groups must be forced out of the plane of the monolayer and can make no contribution to the value of the apparent molecular area. From the dimensions of the parent $\mathrm{Pc}$ based on X-ray studies ${ }^{5}$, the molecule can be approximated as a square of about $10 \AA$ on a side. However, from two-dimensional models which more closely approximate the real contours of the molecule, it is seen that the densest packing of molecular shapes yields a minimum area of about $76 \AA^{2}$, close to the observed value of $80 \AA^{2}$, assuming no contribution from the side chains. The only other reported instance ${ }^{6}$ of a Pc macrocycle oriented parallel to the water surface in its spread monolayer is that of the copper octa(dodecoxymethyl) Pc.

Mixed monolayers of SG-A and methyl eicosanoate were prepared at various mole ratios, and isotherms determined. The isotherms indicated that the two compounds were miscible. Observed values for mixed monolayer areas were less than those calculated on the basis of molecular areas of the pure components and their mole ratios at various concentrations. The mixed monolayer systems were evidently true molecular dispersions rather that separate, discrete mosaic patches of pure constituents.

The surface isotherms determined for SG-A is shown in Fig. 2 and is typical of those seen for all other compounds studied, with the exception of SG-C.

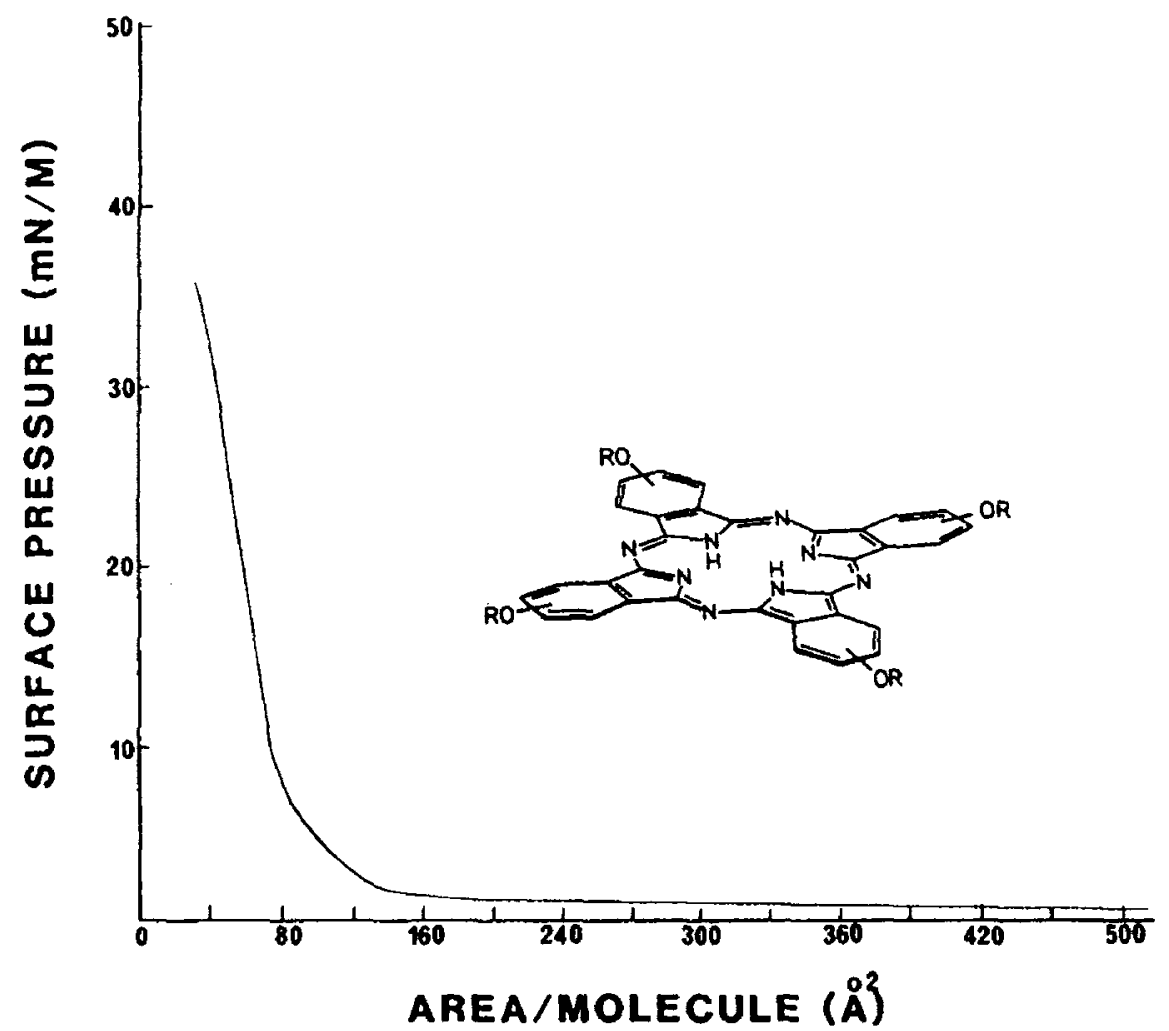

Fig. 2. Surface pressure-area isotherms of SG-A. 


\section{2. $S G-B, S G-25, S G-35$ and $S G-C$}

All these compounds have two $\mathrm{Pc}$ macrocycles linked together by a variety of interconnecting bridges. The allowable number of configurational states varies greatly throughout the series and this is reflected in the observed molecular areas. An assessment of the influence of small variation in molecular structure on the nature of the resulting spread monolayer is thus possible.

These dimeric forms could be of value as surrogates for the chlorophyll "special pair" found in the photosynthetic reaction center. There, light energy is converted into chemical potential after a rapid charge separation. The chlorophyll special pair in vivo is held at a specific orientation and intermolecular distance by interaction with the protein matrix, and the complete structure of the complex is now known?

Pc systems have been extensively studied in the context of their electrocatalytic, photovoltaic and photocatalytic effects. For the case of simple $\mathrm{Fe}^{\mathrm{Il}} \mathrm{Pc}$, it is known that the four-electron reduction of oxygen can be effected. Binuclear species are expected to be more efficient.

\section{3. $S G-B$}

The direct bonding of the rings of SG-B must produce a steric hindrance great enough to prevent the flexibility necessary for coplanar structuring of both macrocycles. However, this direct link also is too short to allow cofacial orientation. The decrease in molecular area at decreased compression rates indicates that a monolayer of most dense packing will not be quickly structured at higher compression rates. The values determined for the apparent area per molecule could be reasonably interpreted as indicating that the system has one macrocycle flat on the water surface with the second ring assuming some oblique angle.

\section{4. $S G-25$}

The intermacrocycle connection in SG-25 affords a much greater flexibility than seen in SG-B, and the possibility that macrocycles would tend to orient cofacially, with the oxygen tending to interact with the water surface. The small molecular area could be interpreted as an indication of edge-on orientation of less than $90^{\circ}$ to the water plane. Alternatively, one macrocycle could be flat on the surface with the second ring forming a bilayer on top of it.

\section{5. $S G-35$}

In this case, no oxygen is present to confer hydrophilicity and the Pc macrocycles are separated at distances even greater than those in SG-25. The possibility for cofacial interaction of the rings is greater, and for this reason the SG-35 monolayer may more closely resemble that of tetra-t-butyl Pc. It appears that the average angle of macrocycle planes to the surface must be near $90^{\circ}$ in order to produce the observed molecular area of only $60 \AA^{2}$, which is twice $10 \AA$ (the Pc edge distance) multiplied by $3 \AA$ (the thickness of the macrocycle).

\section{6. $S G-C$}

Of all the compounds studied, only SG-C produced isotherms which invariably had shoulders indicating a conformational transition state at intermediate film areas 


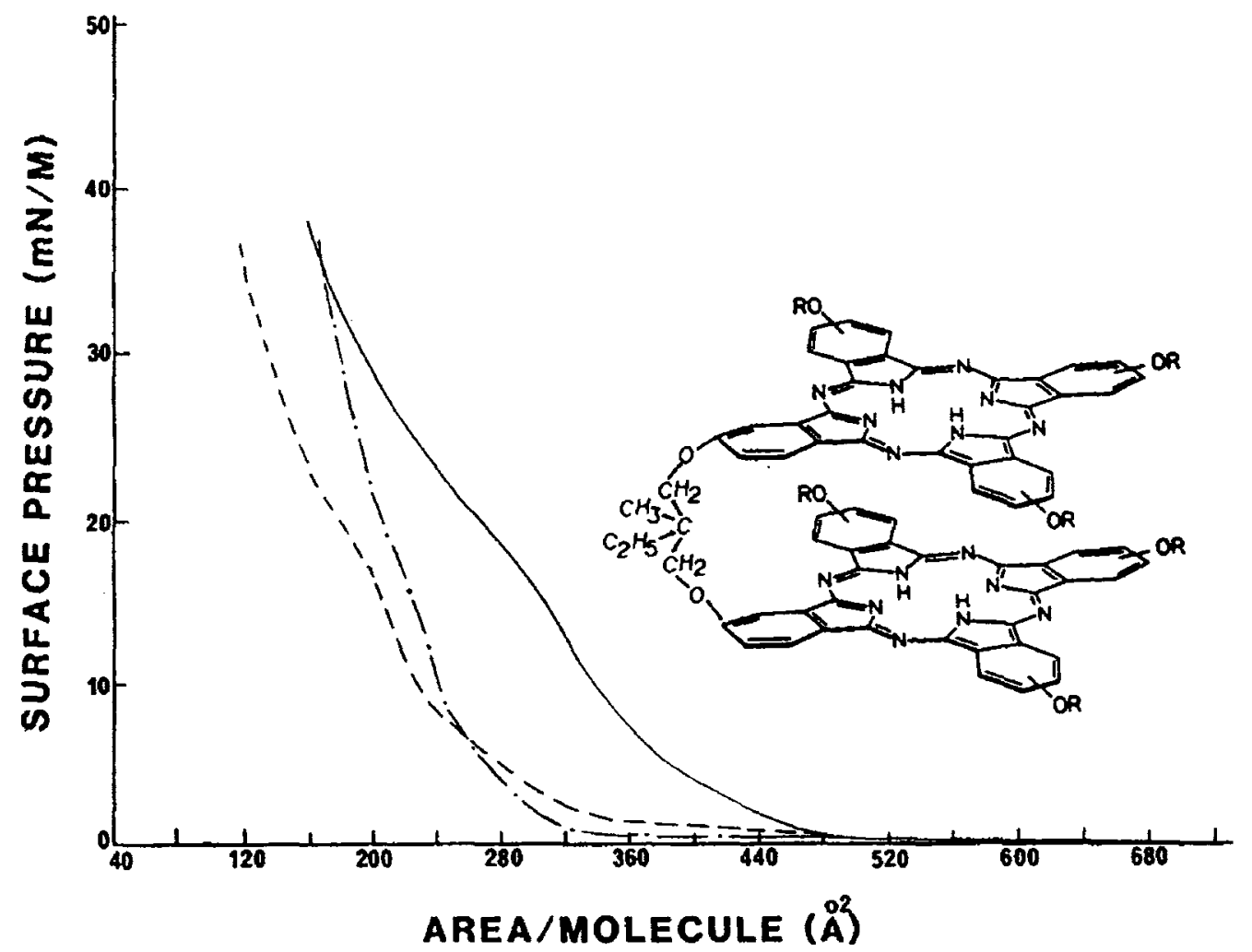

Fig. 3. Surface pressure-area isotherms of $\mathrm{SG}-\mathrm{C}$ at various temperatures: $-1,13.3^{\circ} \mathrm{C}$; - - $22.3^{\circ} \mathrm{C} ;--, 31.6^{\circ} \mathrm{C}$.

(Fig. 3). This could be caused by a more extreme separation of the macrocycles which are now separated by a five member chain. A very large number of configurational states are possible in this structure. A similar shoulder, seen in the pressure region of $20-30 \mathrm{mN} \mathrm{m}^{-1}$, has been reported ${ }^{6}$ in the isotherm of copper octa(dodecoxymeythl) $\mathrm{Pc}^{6}$ and must relate to some restructuring process in the pendant side chains.

The effects of temperature were investigated (Table I); at the lowest temperature a somewhat more pronounced transition region can be seen. An equivalent effect can be seen in terms of the compression rate, the lower compression speed producing a smaller apparent molecular area. The SG-C molecule appears to have a flat orientation on the water surface, a condition favored by the extreme separation of the rings and the presence of the two oxygen atoms.

At present, it is not clear why other compounds studied in this series fail to show evidence of a shoulder in their isotherms.

\section{7. $S G-26 R$}

The linear trimer SG-26R is structurally a polymeric extension of SG-A, and its molecular area of about three times that of SG-A indicates that it, too, is oriented flat on the surface.

\subsection{SG-tet}

The variety of configurational states of this molecule again imply that a 
minimum molecular area may be achieved only at low compression rates. The measured molecular area would require a structure in which two macrocycles are lying flat on the surface, with the additional two oriented above, producing bilayer or other multilayer structures.

\section{CONCLUSIONS}

The monolayer behavior of substituted, multinucleate $\mathrm{Pc}$ structures is given here for the first time. The presence of two or more covalently bonded macrocycles in the same molecule greatly increases the number of configurational states possible and consequently will increase the complexity of events during monolayer compression. Although a number of plausible orientational states can be postulated on the basis of the isotherms measured, considerable additional characterization will be necessary before complete structural details are firmly established. To establish more completely the orientations of these systems as transferred Langmuir-Blodgett films, future studies will include ellipsometry and dichroic measurements.

\section{ACKNOWLEDGMENT}

The financial support of the U.S. Department of Energy, Chemical Sciences Division (DE-FG02-84ER13261), is gratefully acknowledged.

\section{REFERENCES}

1 S. Baker, M. C. Petty, G. G. Roberts and M. V. Twigg, Thin Solid Films, 99 (1983) 53.

2 H. Kuhn, Thin Solid Films, 99 (1983) 1.

D. Möbius, Acc. Chem. Res., 14 (1981) 63.

3 A. W. Snow and N. L. Jarvis, J. Am. Chem. Soc, 106 (1984) 4706.

4 S. M. Marcuccio, P. I. Svirskaya, S. Greenberg, A. B. P. Lever, C. C. Leznoff and K. B. Tomer, Can. J. Chem., 63 (1985) 3057.

S. Greenberg, S. M. Marcuccio, C. C. Leznoff and K. B. Tomer, Synthesis, (1986) 406.

5 C. J. Schramm, R. P. Scaringe, D. R. Stojakovic, B. M. Hoffman, J. A. Ibers and T. J. Marks, J. Am. Chem. Soc., 102 (1980) 6702.

6 D. W. Kalina and S. W. Crane, Thin Solid Films, 134 (1985), 109.

7 J. Deisenhofer and H. Michel, Springer Ser. Chem. Phys., 42 (1985) 94. 